\title{
Unitary Evolution on a Discrete Phase Space
}

\section{Emmanuel G. Floratos}

Physics Department, University of Athens, Athens, Greece

E-mail: mflorato@phys.uoa.gr

\section{Stam Nicolis*}

CNRS-LMPT Tours, Parc Grandmont, 37200 Tours, France

E-mail: stam.nicoliselmpt.univ-tours.fr

We construct unitary evolution operators on a phase space with power of two discretization. These operators realize the metaplectic representation of the modular group $S L\left(2, \mathbb{Z}_{2^{n}}\right)$. It acts in a natural way on the coordinates of the non-commutative 2-torus, $\mathbb{T}_{2^{n}}^{2}$ and thus is relevant for noncommutative field theories as well as theories of quantum space-time. The class of operators may also be useful for the efficient realization of new quantum algorithms.

XXIIIrd International Symposium on Lattice Field Theory

25-30 July 2005

Trinity College, Dublin, Ireland

${ }^{*}$ Speaker. 


\section{Introduction}

Recent progress in M-theory indicates that spacetime itself becomes noncommutative at scales where D-branes play an important role [1,2]. This noncommutativity comes about in a rather natural way because D-branes are charged, gravitational solitons, moving in backgrounds with magnetic flux and their worldvolume acquires non-commutative geometry. What happens is analogous to the Landau problem, where the noncommutativity of the two, real, space coordinates is brought about by the magnetic flux[3]. The strength of the flux provides a measure of non-commutativity. This is the first time where explicit dynamics on non-commutative spacetime has been throughly studied and it has led to a new understanding of Yang-Mills theories (with fluxes) in commutative spacetime as $U(1)$ gauge theories in non-commutative spacetime.

The new insight is the trading of spacetime non-commutativity with the non-commutativity of the gauge group. In general it has been established that Yang-Mills gauge theories (in commutative spacetime) have similar short-distance behavior but different long-distance behavior from noncommutative Yang-Mills theories.

The above facts led people to think again the old idea of discretization of spacetime with cellular structure, which could be described by non-commmutative coordinates. For Minkowski spacetimes care must be taken to use non-commutativity only in the space part, otherwise unitarity in perturbation theory is lost.

More drastic ideas, not coming from D-brane studies, have been advanced by 't Hooft[4] in an effort to reconcile gravity with quantum mechanics. The proposal is that, at scales where gravity becomes strong, it is no longer appropriate to work with continuous variables, but at the classical level, to use discrete labels for spacetime coordinates as well as the dynamical state of the system. Quantum mechanics arises from the superoposition of these classical dynamical states and the mapping of the confi guration and momentum space in wavefunctions on a fi nite-dimensional (and discrete) Hilbert space. Locality in space is lost, since there is a minimal lengthscale and, because of causality, locality is lost in time as well. Hamiltonians are thus non-local and only unitary, one-time-step evolution operators have any meaning.

In this contribution we present the construction of unitary evolution operators on a toroidal phase space with power of two discretiztion. This case could not be included in the previous cases of prime or odd discretization[5] due to the impossibility of defi ning $1 / 2 \bmod 2^{n}$. The solution we present here relies in absorbing these factors and considering twice as many points per phase space direction; We then show that the restriction to half the points is consistent and present the transformation that groups them together in order to ensure a unitary evolution.

We study a model for a discrete and periodic one-dimensional space and, at the same time, discrete and periodic momentum, i.e. a discretized, toroidal, phase space, $\mathbb{Z}_{N} \times \mathbb{Z}_{N}$, on which classical, linear, maps, elements of $S L\left(2, \mathbb{Z}_{N}\right)$, which discretize continuous, $S L(2, \mathbb{R})$ maps on the 2-torus, of unit length, $\mathbb{T}^{2}$, for motions on the rational points, with denominator $N$. Taking $N \rightarrow \infty$ a subset of possible trajectories has a smooth limit and we recover $S L(2, \mathbb{R})$. This discretization has the nice property, that can be transferred to the quantum-mechanical level, of assigning to each $\mathrm{A} \in S L\left(2, \mathbb{Z}_{N}\right)$, a unique, $N \times N$, unitary map, $U(\mathrm{~A})$, which represents faithfully sequences of classical maps that are irreducible representations of $S L\left(2, \mathbb{Z}_{N}\right)$. This can be done for any $N$ odd using as elementary blocks the case $N=p^{n}, p$ prime and, for the general case, $N=p_{1}^{n_{1}} \times \cdots p_{k}^{n_{k}} \cdots$, 
we use the basic property of $S L\left(2, \mathbb{Z}_{N}\right)$,

$$
S L\left(2, \mathbb{Z}_{N}\right)=\otimes S L\left(2, \mathbb{Z}_{p_{k} n_{k}}\right)
$$

In the case $N=2^{n}$, or, more generally, $N$ even, there are constraints imposing periodicity on the unitary matrices $U(\mathrm{~A})$, which restrict the group $S L\left(2, \mathbb{Z}_{N}\right)$ to its normal subgroup

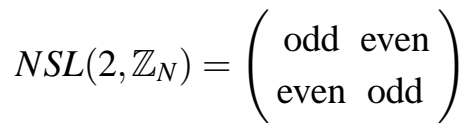

There is an additional subset of $S L\left(2, \mathbb{Z}_{N}\right)$, consisting of elements

$$
A=\left(\begin{array}{cc}
\text { even } & \text { odd } \\
\text { odd } & \text { even }
\end{array}\right)
$$

which, together with the elements of $\operatorname{NSL}\left(2, \mathbb{Z}_{N}\right)$, form a bigger subgroup, which satisfy the periodicity constraints.

In the next section we give the basic defi nitions for the metaplectic representation and we quantize translations and dilatations. Using these as building blocks, it is thus possible to quantize any classical action. We close with a brief discussion of direction of future inquiry.

\section{Unitary Evolution for Translations and Dilatations}

We start with the observation that any matrix A, element of $\operatorname{SL}\left(2, \mathbb{Z}_{N}\right)$, may be written as

$$
\underbrace{\left(\begin{array}{ll}
a & b \\
c & d
\end{array}\right)}_{\mathrm{A}}=\underbrace{\left(\begin{array}{cc}
1 & b d^{-1} \\
0 & 1
\end{array}\right)}_{\mathrm{L}\left(b d^{-1}\right)} \underbrace{\left(\begin{array}{cc}
d^{-1} & 0 \\
0 & d
\end{array}\right)}_{\mathrm{D}(d)} \underbrace{\left(\begin{array}{cc}
1 & 0 \\
c d^{-1} & 1
\end{array}\right)}_{\mathrm{R}\left(c d^{-1}\right)}
$$

i.e. in terms of translations and dilatations in phase space. We note that obstructions to this construction may appear, if $d$ is not invertible mod $N$. For $N=2^{n}$ this means, in particular, that $d$ should be odd. We shall try to construct the unitary operator, $U(\mathrm{~A})$ from the corresponding unitary operators

$$
U^{\mathrm{L}}(x) \equiv U(\mathrm{~L}(x)) U^{\mathrm{D}}(d) \equiv U(\mathrm{D}) U^{\mathrm{R}}(y) \equiv U(\mathrm{R}(y))
$$

For any discretization $N$ it is possible to find operators $J_{r, S}$ that generate the Heisenberg-Weyl group. They are given in terms of the clock and shift operators $Q$ and $P$ by

$$
J_{r, s}=\omega_{N}^{r s / 2} P^{r} Q^{s}
$$

where $\omega_{N} \equiv \exp (2 \pi \mathrm{i} / N)$. In the basis where the clock operator $Q$ is diagonal the matrix elements are given by

$$
P_{k, l}=\delta_{k-1, l} Q_{k, l}=\omega_{N}^{k} \delta_{k, l}\left[J_{r, s}\right]_{k, l}=\delta_{k-r, l} \omega_{N}^{s(k+l) / 2}
$$

where $k, l=0,1, \ldots, 2^{n}-1$. We already notice the $1 / 2$ factors that need to be defi ned seperately, when $N=2^{n}$. The unitary operators $U(\mathrm{~A})$ need to satisfy two requirements: 
- They realize a group representation: for any two operators, $A, B \in S L\left(2, \mathbb{Z}_{2^{n}}\right)$ we have

$$
U(\mathrm{~A} \cdot \mathrm{B})=U(\mathrm{~A}) \cdot U(\mathrm{~B})
$$

- They realize the metaplectic representation: for any point, $(r, s)$, of the classical phase space

$$
U(\mathrm{~A}) J_{r, s} U(\mathrm{~A})^{-1}=J_{(r, s) \mathrm{A}}
$$

In this section we shall sketch the construction for $U^{\mathrm{L}}(x), U^{\mathrm{R}}(y)$ and $U^{\mathrm{D}}(d)$. Details may be found in the paper[7].

We fi rst consider a larger space, of $2^{n+1}-1$ points, i.e. $\operatorname{SL}\left(2, \mathbb{Z}_{2^{n+1}}\right)$. In this space we defin ne the matrix elements of $J_{r, s}$ as

$$
\left[J_{r, s}\right]_{k, l}=\delta_{k-r, l}^{(n+1)} \omega_{n+2}^{s(k+l)}
$$

where $\omega_{n} \equiv \exp \left(2 \pi \mathrm{i} / 2^{n}\right)$ and the superscript on the Kronecker delta indicates that the operation is performed $\bmod 2^{n+1}$. Note that we have absorbed the $1 / 2$ factor in the order of the root of unity.

However the "physical" points correspond to the even values of the indices $r, s, k, l$ and, thus the "physical" sub-space is, indeed, $2^{n}$-dimensional.

It is possible to show that the following operators realize a group representation and the metaplectic reprsentation:

$$
\begin{gathered}
{\left[U^{\mathrm{L}}(x)\right]_{k, l}=\frac{1+(-)^{k}}{2} \omega_{n+2}^{x k^{2}} \delta_{k, l}^{(n+1)}} \\
{\left[U^{\mathrm{D}}(d)\right]_{k, l}=\delta_{k, d l}^{(n+1)}} \\
{\left[U^{\mathrm{R}}(y)\right]_{k, l}=\left[F^{-1} U^{\mathrm{L}}(-y) F\right]_{k, l}=\frac{1}{2^{n+1}} \sum_{m=0}^{2^{n}-1} \frac{1+(-)^{l+k}}{2} \omega_{n}^{-m^{2} x+m(l-k)}}
\end{gathered}
$$

where $F$ is the Fourier Transform operator,

$$
F_{k, l}=\frac{\omega_{n+1}^{k l}}{\sqrt{2^{n+1}}}
$$

Here the indices $k, l=0,1, \ldots, 2^{n+1}-1$-however the projector

$$
\frac{1+(-)^{k}}{2}
$$

projects on the even values, $k, l=0,2,4, \ldots, 2^{n+1}-2$. Similarly, when checking the metaplectic representation, eq. (2.5), the indices $r, s=0,2,4, \ldots, 2^{n+1}-2$. That this is consistent may be deduced from the fact that the points of the classical phase space ( $r=$ even, $s=$ even) are transformed among themselves by a matrix $A \in S L\left(2, \mathbb{Z}_{2^{n}}\right)$. We thus may check that the evolution is unitary on this sub-lattice. However, since we have introduced a projector, unitarity is spoiled on the original lattice. Remains then to construct an operator that rearranges the sites in such a way as to render 
unitarity manifest on the even sub-lattice. Such an operator is the bit reversal operator used in the Fast Fourier Transform[6, 7]!

We thus may conclude that the general classical action $\mathrm{A} \in S L\left(2, \mathbb{Z}_{2^{n}}\right)$, with the element $d$ odd, may be consistently quantized through an embedding in a space of twice as many points. Details and proofs may be found in [7].

\section{Conclusions and Outlook}

We have presented the construction of unitary evolution operators that consistently quantize the classical action on phase spaces of power of two discretization. This completes the program of papers[5] and may open the way for new quantum algorithms based on operators that are more general than the Fourier Transform. Furthermore the dimensionality considered here may be useful in the description of systems with fermionic degrees of freedom.

\section{References}

[1] T. Banks, W. Fischler, S. Shenker and L. Susskind, M-Theory as a Matrix Model: A Conjecture, Phys. Rev. D55 (1997) [hep-th/ 9610043$]$;

[2] A. Connes, M. R. Douglas and A. Schwarz, Noncommutative Geometry and Matrix Theory: Compactification on Tori, JHEP 02 (1998) 003 [hep-th/9711162]; B. Pioline and A. Schwarz, Morita equivalence and T-duality (or B versus $\Theta$ ), JHEP 08 (1999) 021, [hep-th/ 9908019$] \mathrm{N}$. Seiberg and E. Witten, String Theory and Noncommutative Geometry, JHEP 09 (1999) 032, [hep-th/9908142];

[3] A. Connes, Noncommutative Geometry, Academic Press (1994).

[4] G. 't Hooft, Quantization of Discrete Deterministic Theories by Hilbert Space Extension, Nucl. Phys. B342 (1990) 471; G. 't Hooft, K. Isler and S. Kalitzin, Quantum Field Theoretic Behavior of a Deterministic Cellular Automaton, Nucl. Phys. B386 (1992) 495; G. 't Hooft, Determinism amd Dissipation in Quantum Gravity, [hep-th/0003005].

[5] G. G. Athanasiu and E. G. Floratos, Coherent States in Finite Quantum Mechanics, Nucl. Phys. B425 (1994) 343.G. G. Athanasiu, E. G. Floratos and S. Nicolis, Holomorphic Quantization on the Torus and Finite Quantum Mechanics, J. Phys. A: Math. Gen. 29 (1996) 6737, [ [ hep-th/ 9509098 ]; G. G. Athanasiu, E. G. Floratos and S. Nicolis, Fast Quantum Maps, J. Phys. A: Math. Gen. 31 (1998) L655, [math-ph/9805012]; E. G. Floratos and S. Nicolis, An SU(2) Analog of the Azbel-Hofstadter Hamiltonian, J. Phys. A: Math. Gen. 31 (1998) 3961, [hep-th/9508111].

[6] W. Press, B. P. Flannery, S. A. Teukolsky and W. T. Vetterling, Numerical Recipes in C, Cambridge University Press (1992).

[7] E. G. Floratos and S. Nicolis, Unitary Evolution on the $\mathbb{Z}_{2^{n}} \times \mathbb{Z}_{2^{n}}$ Phase Space [hep-th/ 0505229 ] 